\title{
Téoros
}

Revue de recherche en tourisme

\section{Les expositions commerciales à Montréal : un secteur en développement}

\section{François Cadoret}

Volume 10, numéro 3, novembre 1991

Destination Montréal : d’hier à demain

URI : https://id.erudit.org/iderudit/1079198ar

DOI : https://doi.org/10.7202/1079198ar

Aller au sommaire du numéro

Éditeur(s)

Université du Québec à Montréal

ISSN

0712-8657 (imprimé)

1923-2705 (numérique)

Découvrir la revue

Citer cet article

Cadoret, F. (1991). Les expositions commerciales à Montréal : un secteur en développement. Téoros, 10(3), 25-29. https://doi.org/10.7202/1079198ar d'utilisation que vous pouvez consulter en ligne.

https://apropos.erudit.org/fr/usagers/politique-dutilisation/ 
Cet article résume une étude effectuée par Price Waterhouse pour le compte de la CIDEM (Ville de Montréal)en 1990. Les résultats présentés ci-dessous ne concernent qu'une partie de cette étude.

Les expositions commerciales ${ }^{(1)}$ représentent une variable importante de la stratégie de marketing de plusieurs entreprises oeuvrant dans une multitude de secteurs d'activité économique. En effet, il est possible pour une entreprise de contacter ses clients actuels et potentiels par différents moyens, mais les expositions commerciales présentent toutefois plusieurs avantages et ce, tant pour l'entreprise qui vend des produits ou services que pour celle qui les achète, ou enfin pour le consommateur. Parmi les avantages majeurs, notons:

- la possibilité de rencontreren un même lieu et dans un court laps de temps un nombre important de clients actuels;

- la possibilité de rencontrer et de solliciter sur une courte période de temps un nombre important de clients potentiels connus, mais également de prendre contact avec des clients totalement nouveaux;

- la possibilité de se familiariser avec l'offre de ses concurrents;

- la réduction du coût de visites aux clients sur une base individuelle;

- la possibilité de se tenir au courant des innovations et des développements propres a l'industrie.

Cette stratégie de marketing est utilisée par les entreprises au Canada comme ailleurs dans le monde. Ce phénomène semble toutefois relativement plus important en Europe qu'en Amérique du Nord, dans le cas des expositions commerciales pour l'industrie. En effet, la participation des entreprises européennes à des expositions

Monsieur Franços Cadoret est directeur au sein des services conseils en marketing dans le domaine du tourisme d'accueil chez Price Waterhouse. commerciales représente une composante proportionnellement plus importante de la stratégie de marketing et de vente que ce que l'on retrouve au niveau des entreprises nord-américaines. De façon plus précise, mentionnons que la portion du budget total de promotion des vente quiestaccordée à la participation aux expositions est supérieure à $60 \%$ en Europe alors qu'elle est inférieure à $40 \%$ en Amérique du Nord. De plus, la durée de ce type d'expositions en Europe est généralement beaucoup plus longue qu'en Amérique du Nord, ce qui explique en partie l'importance plus grande du budget accordé à la participation aux expositions.

Il est bien connu par ailleurs que la tenue d'expositions commerciales pourl'industrie etpour le consommateur présentel'avantage substantiel pour les villes hôtes de générer des retombées économiques intéressantes: de plus, elle favorise le développement de 1 'industrie régionale concernée par l'évênement. Les retombées économiques sont toutefois plus importantes lorsque les expositions s"adressent principalement à l'industrie plutôt qu'aux consommateurs. Cet écart est dû au fait que ce type d'exposition vise une clientèle provenant d'un rayonnement géographique plus large. En ce sens, il est légitime et approprié pour Montrêal de se doter d'une stratégie de développement visant à favoriser la tenue d'expositions commerciales et, de façon plus particulière, d'expositions pour l'industrie.

\section{Caractéristiques de l'offre internationale au niveau des expositions commerciales}

Pour les fins de cet article, le vocable offre signifie l'ensemble des expositions commerciales qui sont présentées à uneclientèle d'affaires dans les cinq pays étudiés (Canada, États-Unis, Allemagne, France et Royaume-Uni).

Avant de présenter les caractéristiques de l'offre internationale en ce qui a trait aux expositions commerciales, il est opportun de bien délimiter les catégories d'expositions qui ont ếté retenues aux fins de l'analyse. Celles-ci sont:

- les expositions à caractère commercial qui s'adressent uniquement à une clientèle corporative;

- les expositions à caractère commercial qui s'adressent à une clientèle publique et corporative.

Soulignons, de plus, que les conventions et les congrès qui ne sont pas associés à un caractère commercial via un nombre important d'exposants n'ont pas été retenus.

Les systèmes de classification des expositions commerciales utilisés au Canada, aux États-Unis et en Europe ne sont pas identiques. En ce sens, Price Waterhouse a dû créerun système de classification harmonisé afin de présenter le portrait des expositions de façon comparable entre les trois régions géographiques.

Les faits saillantsqui ressortent de l'analyse des caractéristiques de l'offre internationale sont les suivants.

Les secteurs les plus représentés ${ }^{(2)}$.

Au Canada:

- $20 \%$ des expositions canadiennes les plus fréquentes appartiennent au secteur du cadeau, $12 \%$ sont reliées au secteur de l'ameublement et de la décoration intérieure et, enfin, chacun des autres secteurs ne représente qu'une faible proportion de l'ensemble des 50 plus importantes expositions.

Aux États-Unis:

- Parmi les 100 plus grosses expositions commerciales aux États-Unis, l'habillement/vêtement est le secteur le mieux représenté avec $12 \%$ des expositions.

- Les secteurs du cadeau (8\%), du matériel de sport et de récréation (7\%), de 
la machinerie de fabrication ( $6 \%$ ) ainsi que de la construction ( $6 \%$ ) sont aussi représentés de façon importante.

- Plusieurs autres secteurs suivent avec $4 \%$ chacun. Ce sont l'ameublement et la décoration intérieure, les autos et camions, les bateaux, leséquipements de bureau, l'habitation, les ordinateurs, la publicité/marketing et finalement, les sciences.

- Les autres secteurs représentent un pourcentage très faible de l'ensemble des expositions.

\section{Eu Europe:}

- L'agriculture/élevage, l'ameublement et la dếcoration intérieure, les autos et camions, la construction, la machinerie de fabrication ainsi que la transformation des aliments et la distribution accaparent la plus grande part des plus importantes expositions commerciales des pays analysés avec $5 \%$ chacun, soit $30 \%$ de l'ensemble.

- D'autres secteurs sont assez bien représentés avec $4 \%$ chacun. Ce sont les articles ménagers, les équipements de bureau, l'électrique et l'électronique. Les jouets et passe-temps composent, quant à eux, $3 \%$ des 60 expositions les plus importantes de ces trois pays.

- L'Allemagne est le maître d'oeuvre dans le secteur de Ia machinerie de fabrication et des equipements de bureau alors que le Royaume-Uni se concentre sur l'agriculture/élevage, la transformation d'aliments et la distribution ainsi que les jouets et passetemps.

- La France se spécialise quant à elle dans les articles ménagers, l'ameublement et la décoration intérieure.

Les principales caractéristiques des expositions:

Au Canada:

Seulement deux expositions ont une superficie nette supérieure à 300000 pi. ca.; $22 \%$ ont une superficie supérieure ou égale à $200000 \mathrm{pi}$. ca. et enfin, $34 \%$ ont une superficie se situant entre 100000 et 200000 pi. ca..

- Une seule exposition a plus de 1000 exposants, $20 \%$ des 50 expositions ont de 500 a 1000 exposants et $54 \%$ ont entre 250 et 499 exposants.

- Le prix au pied carré varie énormément selon les services qui sont inclus. Le minimum se situe à 3,15 st le maximum a $20,50 \$$. Cinquante pour cent se retrouvent entre $5 \$$ et 10 et $33 \%$ sont entre $15 \$$ et $20 \$$.

- La plus grosse exposition canadienne serait au 24 e rang aux Etats-Unis en termes de superficie.

Aux États-Unis:

- Les 100 plus grosses expositions aux États-Unis couvrent des superficies nettes allant de 170000 à 879502 pi. ca.. Trente-six expositions ont une super ficie superrieure à 300000 pi ca.; parmicelles-ci, 16 expositions ontplus de 500000 pi. ca.

- Le nombre d'exposants varie de 90 à 3000. Quarante-six pour cent des expositions attirent plus de $1000 \mathrm{ex}$ posants. Les 40 plus grosses attirent, en moyenne, 1100 exposants.

- Les frais d'exposition peuvent aller jusqu'à 30 \$ US le pied carrế. La moyenne se situe toutefois autour de $12,30 \$$ le pied carré.

\section{En Europe:}

- Les grosses expositions europenenes sont nettement supérieures aux expositions nord-américaines en terme de superficie. Les plus grosses expositions des trois pays analysés ont une superficie nette allant de 200000 à 3 $658253 \mathrm{pi}$. ca.. Trente-cinq pour cent des 60 expositions analysées dépassent la superficie de 1000000 de pi. ca. En Allemagne, $80 \%$ des 20 plus grosses expositions dépassent cette superficie, comparativement à $15 \%$ pour la France et $10 \%$ pour le Royaume-Uni.

- Quant au nombre d'exposants, il varie de 215 à 8535. En moyenne, cela représente 2761 exposants pour les 20 plus grosses expositions de I'Allemagne, 1266 exposants pour la France et 858 exposants pour le Royaume-Uni.

\section{Identification des secteurs prometteurs pour la tenue d'expositions commerciales}

\section{La méthodologie}

Les systemes de classification des secteurs d'activité économique pour lesquels se tiennent des expositions commerciales sont différents au Canada, aux États-Unis et en Europe. Nous avons donc utilisé le système de classification que Price Waterhouse a développé dans le but d'effectuer une sélection au niveau de l'offre d'abord, et de la demande ensuite. En effet, même si plusieurs secteurs d'activité sont intéressants pour la tenue d'expositions commerciales, il importe de définir un ordre de degré d'intérêt des secteurs pour lesquels il serait plus opportun de déployer des efforts.

\section{La sélection au niveau de la demande}

Pour faire la sélection quant à la demande, trois indices ont été créés et compilés pour les 28 secteurs retenus du côte de l'offre. Ces indices sont: le poids économique du secteur, le degré de croissance du secteur et la répartition des entreprises dans le secteur selon leur taille.

De plus, Price Waterhouse a Également intégré dans son analyse deux éléments supplémentaires soit: les priorités gouvernementales québécoises en matière de développement économique par secteur et l'éloignement des territoires considérés et la propension estimée des entreprises qui participeront éventuellement aux expositions à se déplacer vers le Québec.

Les figures 1 et 2 donnent un aperçu des résultats obtenus.

Finalement, un autreélément très important que nous avons considéré lors de l'analyse est la situation en ce qui a trait aux expositions déjà tenues à Montréal de mểne que dans d'autres villes potentiellement concurrentes à Montréal.

\section{Les résultats}

En combinant les résultats de ces différentes étapes d'analyse au niveau de l'offre et 
FIGURE 1

Matrice du potentiel relatif des secteurs d'activités économiques en matière d'expositions commerciales (catégorie industrielle et service)

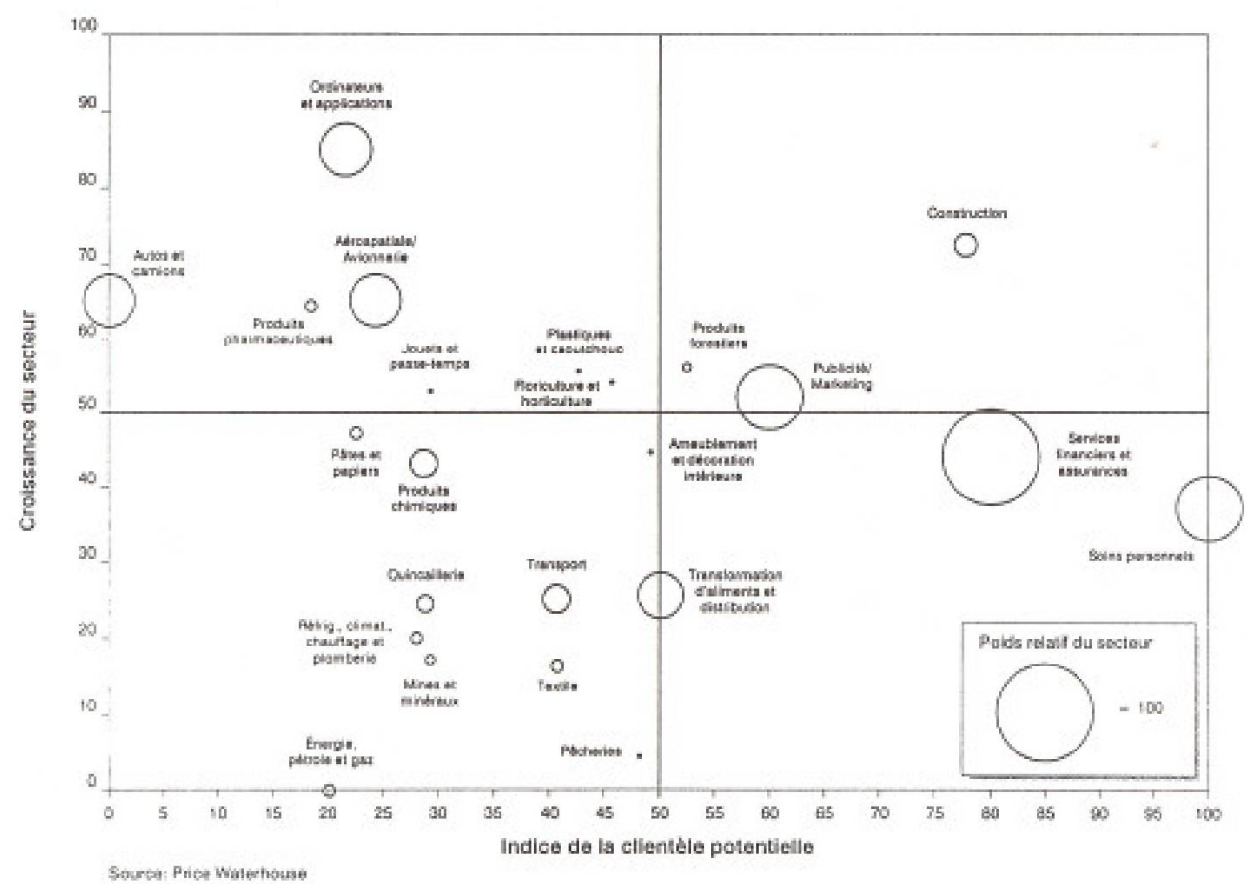

FIGURE 2

Positionnement des secteurs analysés (poids du secteur vs clientèle potentielle)

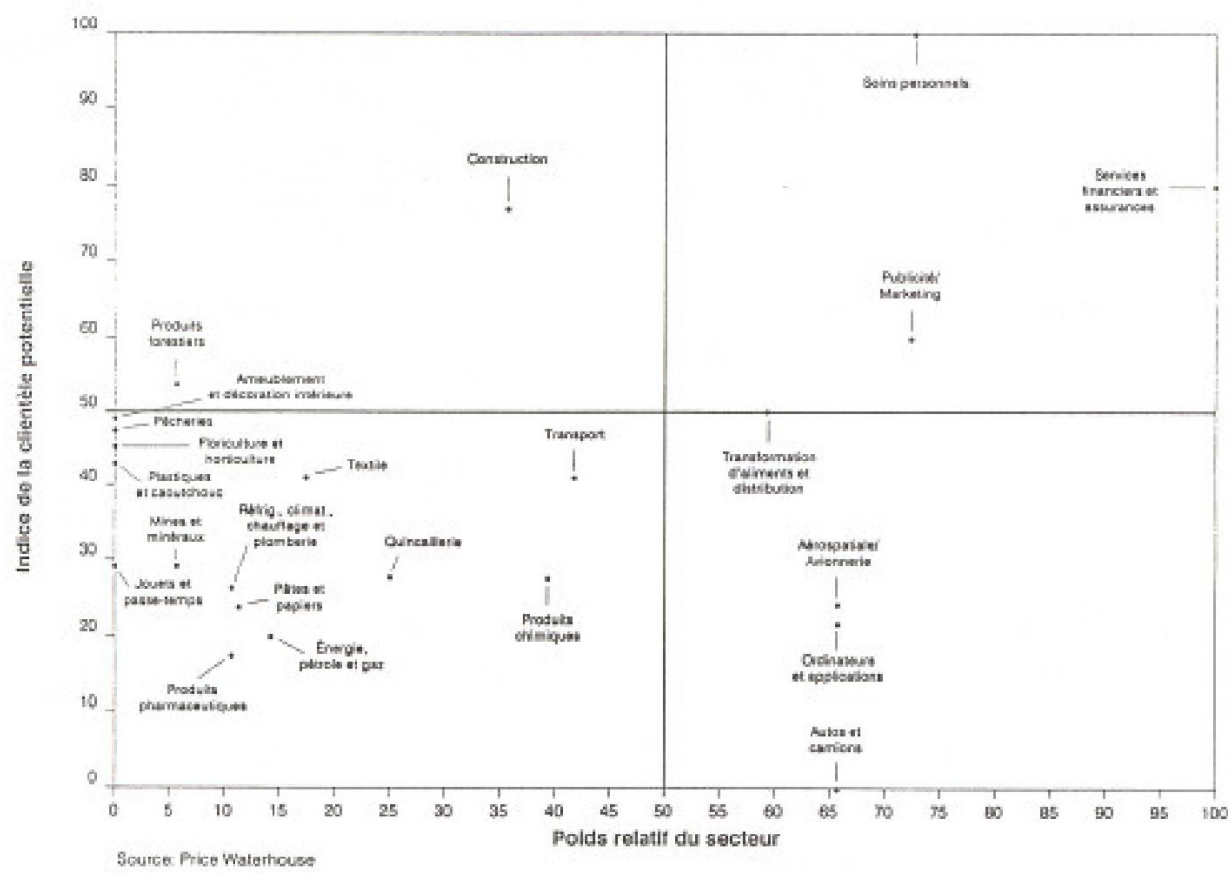

27 TÉonos - Yol 10, no 3, nowmbres 1991 
de la demande d'un point de vue montréalais, québécois, canadien, américain et enropéen, Price Waterhouse a pu identifier deux grandes catégories d'options àctudier pour Montréal soit, d'une part, le lancement de nouvelles expositions et, d'autre part, des incitatifs visant à augmenter la taille d'expositions existant đéjà à Montréal. Le tableau 1 présente ainsi ces résultats.

\section{Les forces et les faiblesses perçues de la Ville de Montréal comme lieu pour la tenue d'expositions commerciales et spécialisées}

Cette section rassemble et résume les opinions et les commentaires émis par les divers intervenants de l'industrie rencontrés: responsables de salles d'exposition, promoteurs d'expositions, etc. Il ne s'agit donc en aucun cas de jugements de valeur.

\section{Les faiblesses de Montréal}

D'un point de vue général:

- Le manque de définition précise du produit touristique montréalais;

- Le climat d'incertitude politique réel ou perçu qui nuit d'une certaine maniêre au développement des expositions commerciales.

- Lasituation géographiquede Montréal (extrême Nord-Est) par rapport aux autres villes importantes du Nord-Est de l'Amérique du Nord.

- La coordination non optimale des efforts de promotion des hôtels avec 1'OCTGM et le ministère provincial du Tourisme.

- La peur chez les Américains de ne pouvoir se faire comprendre en anglais.

Par rapport à Toronto:

- L'espace restreint pour les expositions commerciales par rapport à la principale ville concurrente la plus proche dans le centre du Canada.

- La fréquence et la diversité de la provenance des vols.

\section{TABLEAU 1}

Options à étudier au niveau du potentiel de développement supplémentaire en matière d'expositions commerciales à Montréal
Secteurs

\section{Aérospatiale/Avionnerie}

Ameublement et

Amusement

Construction

Enviromement

Jouets et passe-temps

Mines et minéraux

Ordinateurs at applications

Pătes et papiers

Pêcheries

Plastiques et caoutchouc

Produits chimiques

Produits forestiers

Produits pharmaceutiques

Publicite/Marketing

Quincaillerie

Robotique

Sciences

Soins personnels

Services financiers

Transport

Source: Price Waterhouse décoration intériẹure
Nouvelles
expositions
Croissance des expositions existantes

\section{TABLEAU 2}

La superficle disponible* (pleds carrés) dans les principales salles pour la tenue d'expositions à Montréal et à Toronto

\begin{tabular}{llll} 
Montréal & \multicolumn{3}{l}{ Toronto } \\
\hline Palais des congrès & 145000 & $\begin{array}{l}\text { Toronto International } \\
\text { Center }\end{array}$ & 375000 \\
Place Bonaventure & 255000 & $\begin{array}{l}\text { Metro Toronto } \\
\text { Convertion Center }\end{array}$ & 200000 \\
Stade Olympique & 500000 & Exhibition Place & 1000000 \\
\hline TOTAL: & 900000 & TOTAL: & 1575000 \\
& & Incluant le SkyDome & 1854000
\end{tabular}

- Surface brute.

Source: Price Waterhouse 
- Le nombre restreint d'hôtels à forte capacité d'hébergement.

- Le degré de vieillissement général du parc hôtelier.

- Le faible degré de concertation entre tous les intervenants dans le but de développer les expositions commerciales.

Par rapport à d'autres villes américaines:

- Le fort taux de taxation canadien sur les produits de consommation courante (ex.: alcool, essence, cigarettes).

- L'ampleur du degré de concertation entre tous les intervenants dans le but de développer les expositions com= merciales.

- La perte de vitesse de la perception positive de l'hospitalitế québếcoise.

- Le coüt du billet d'avion.

\section{Les forces de Montréal}

- L'intérêt et la curiosité générés par la culture francophone et le bilinguisme.

- La possibilité de se retrouver dans une ville dont l'ambiance est euro-américaine tout en étant très sécuritaire.

- La présence d'un certain nombre d'hôtels de classe supérieure.

- La plus grande flexibilitế d'accès à la main-d'oeuvre pour les promoteurs étant donné le degré de syndicalisation moins développé qu'à Toronto et qu'ailleurs en Amérique du Nord.

- La qualité reconnue de la restauration montréalaise.

- La possibilité d'offrir des services en français eten anglais, ce qui se veut un avantage pour des expositions canadiennes et intemationales.

\section{La situation quant aux superficies disponibles}

Toronto se veut évidemment un des principaux concurrents de Montréal pour la tenue d'expositions commerciales. Il est donc appropriế de comparer les superficies disponibles et consacrées à cet effet entre les deux villes. Le tableau 2 présente cette information.

Toronto offre une superficie disponible equivalent à 1.8 fois ou 2.1 fois celle de Montréal, si l'on tient compte ou non de la superficie du SkyDome. De plus, les intervenants de l'industrie s'accordent à dire que la nature et la capacité des infrastructures actuelles ne seront pas adéquates pour concrétiser les possibilités de développement de l'industrie des expositions commerciales à Montréal.

En effet, la Place Bonaventure est pratiquement occupée à $100 \%$; le Palais des congrès a un taux d'occupation d'environ $60 \%$ mais celui-ci se destine plutôt à la tenue de congrès; et, finalement, le calendrier du Stade Olympique n'offre que peu de flexibilité pour la tenue d'expositions commerciales. Il faut se rappeler que certaines expositions tenues à Montréal manquent déjà d'espaces sur les sites qu'elles occupent. Sur la base de ces constatations, il apparait donc clairement qu'il existe un besoin d'espace supplémentaireà Montréal.

\section{Conclusion}

L'analyse effectuée en 1990 concernant le secteur des expositions commerciales et spécialisées démontre clairement qu'il existe un fort potentiel de développement dans ce domaine. Cependant, les constats suivants s'imposent:

- Le développement des expositions commerciales à Montréal n'est pas une préoccupation majeure des intervenants privés, publics et parapublics actifs au sein de cette industrie, exception faite de certains promoteurs d'expositions.

- Il n'existe pas réellement de concertation entre les intervenants afin de développer l'industrie des expositions commerciales à Montréal.

- Il existe des possibilités de croissance pour les expositions commerciales tenues actuellement à Montréal de même que des possibilités de développement de nouvelles expositions commerciales à Montréal mais la nature et la capacité des infrastructures actuelles ne seront pas adéquates pour concrétiser ces possibilités de dévêloppement.

Compte tenu de ces constatations, il est clair que Montréal devra mobiliser les forces vives de ce secteur d'activités afin d'en arriver à développer celui-ci de façon concertée et ainsi se mettre en meilleure position sur l'échiquier international. De plus, Montréaldevra, en fonction de lacroissance de nouvelles expositions et/ou de celles déjà existantes, faire en sorte qu'il y ait adéquation entre la capacité des infrastructures et le potentiel d'exposition.

\section{Notes et références}

(1) Afin d'alléger le texte, nous utiliserons dans cet article l'expression wexpositions commerciales. plutöt que rexpositions commerciales et specialiseses.

12) En termes de nombre d"expositions, 